\title{
Uma Proposta para o Ensino de Mecânica Quântica
}

\author{
Ileana Maria Greca, Marco Antonio Moreira, Victoria E. Herscovitz \\ Instituto de Física, UFRGS, \\ Caixa Postal 15051, 91501-970, Porto Alegre, RS
}

Recebido em 27 de Abril de 2001. Aceito em 22 de Outubro de 2001.

\begin{abstract}
Em decorrência dos avanços da tecnologia quântica, como fazer para que estudantes de cursos de Ciências Exatas e Engenharia compreendam melhor conceitos centrais da Mecânica Quântica temse convertido em um tema atual de pesquisa na área de ensino de Ciências em nível internacional. Neste trabalho apresentamos a fundamentação teórica de uma proposta didática que aponta neste sentido e os resultados da sua implementação em cursos de Engenharia.
\end{abstract}

\begin{abstract}
As a consequence of the advances of quantum technology, how to help science and engineering majors to get a better undersctanding of key quantum mechanical concepts has became a contemporary research topic in science teaching at international level. In this paper we present the theoretical framework of a didactical approach in this direction as well as the results of its implementation with engineering students.
\end{abstract}

\section{Introdução}

A compreensão dos estudantes sobre conceitos de Mecânica Quântica, o aprimoramento do seu ensino em nível universitário e sua incorporação no ensino médio têm se convertido em temas atuais na pesquisa em Ensino de Ciências. Ainda que até hoje as pesquisas publicadas a este respeito sejam escassas [1], em comparação com outras áreas como a Mecânica ou o Eletromagnetismo, nos últimos anos o número de trabalhos apresentados a este respeito (antes restritos principalmente a modelos atômicos) tanto em congressos e conferências, nacionais e internacionais, como nas revistas da área tem crescido notavelmente [2].

Demais dizer que este interesse é muito justificado. Em parceria com a Relatividade, a Mecânica Quântica é a grande estrela do século XX. Base de sustentação da física nuclear, atômica, molecular e do estado sólido, da física das partículas elementares e da luz, seus impactos práticos atingem hoje as mais variadas aplicações, beneficiando até campos de praticidade imediata como as Ciências da Saúde e as Engenharias. Mais ainda, os desenvolvimentos recentes na miniaturização eletrônica e na nanotecnologia têm introduzido, até no mundo dos negócios, dispositivos que somente podem ser apreciados a partir dos princípios da Mecânica Quântica [3]. Se até agora este conhecimento estava reservado aos estudantes de Física e de Química, parece inevitável que a maioria dos profissionais deste novo século deva ter um conhecimento a este respeito bem mais aprofundado que o necessário até então.

Certamente, ensinar Mecânica Quântica não é uma tarefa fácil. Seus princípios fogem da visão clássica de mundo que possuímos, fazendo que a maioria deles levem a conseqüências que resultam "antiintuitivas". Assim, as implicações resultantes de conceitos como os de superposição de estados, princípio de incerteza ${ }^{1}$, dualidade onda-partícula, distribuição de probabilidades e não localidade continuam até hoje provocando acalorados debates, sendo alvo de críticas até mesmo daqueles que contribuíram a moldá-la.

Por outra parte, a abordagem tradicional das disciplinas introdutórias para os cursos de Ciências Exatas salienta aspectos históricos, que apelam mais para as características clássicas dos sistemas do que para as quânticas, e dos conceitos quânticos antes enunciados, os estudantes recebem, sobretudo, informações na forma de equações ${ }^{2}$, com pouco vínculo com a fenomenologia. Além disto, a forma como ela é apresentada defronta os estudantes com uma série de fatos cuja racionalidade termina resultando duvidosa [4], em contraposição ao ensino das áreas da Física Clássica, que salienta o raciocínio lógico e preciso que caracteriza a "racionalidade" física. Como conseqüência disto, a Mecânica Quântica é considerada como matéria difícil, com poucas ligações com o mundo real e até "esotérica",

\footnotetext{
${ }^{1}$ Ainda que o conceito de incerteza esteja associado também ao conceito clássico de ondas, adquire outro significado no contexto da Mecânica Quântica, sendo estendido a qualquer par de observáveis não simultâneos relativos aos objetos quânticos.

${ }^{2}$ Nos cursos introdutórios, o formalismo não é apresentado de forma integral e os estudantes recebem, em geral, algumas fórmulas isoladas.
} 
sendo seus conceitos não compreendidos pelos estudantes [5-7]. Em resumo, as abordagens típicas destes cursos não criam condições para que os alunos apreendam a nova forma de perceber os fenômenos decorrente dos princípios da Mecânica Quântica e cursos subseqüentes, mais técnicos, que não discutem as concepções em que se baseiam os enunciados da teoria, também não conseguem fazer isso [6],[8].

Estes fatores conduzem a que o desenvolvimento de estratégias didáticas que favoreçam a aprendizagem significativa da Mecânica Quântica se tornem temas de pesquisa relevante. Neste trabalho apresentamos uma proposta que foi implementada no IF-UFRGS durante os semestres letivos 1999/1 e 2, em três turmas da disciplina de Física IV (onde estes conteúdos são tradicionalmente introduzidos) das carreiras de Engenharia e Química, cujo objetivo foi, fundamentalmente, auxiliar os estudantes a visualizar fenômenos microscópicos a partir dos princípios da Mecânica Quântica. Os resultados que apresentamos, no entanto, são os obtidos na primeira implementação da mesma (estudo piloto, primeiro semestre de 1999).

\section{Fundamentação da proposta em termos de aprendizagem}

As pesquisas desenvolvidas pelo Grupo de Ensino do IF-UFRGS nos últimos anos têm-nos levado a considerar, como indica Feyerabend [9], que certas teorias científicas - e em particular as físicas - possuem determinadas características próprias da linguagem natural e, por isso, para poder aprendê-las é preciso aprender novas relações de percepção e conceituais, trazendo à luz concepções ocultas nos significados dos seus enunciados. O referencial teórico com o qual trabalhamos foi a teoria dos modelos mentais de Johnson-Laird [10]; [11]. Segundo ela, um modelo mental é uma representação interna que atua como um análogo estrutural de situações ou processos. Sua função é a de dar conta do raciocínio dos indivíduos tanto quando tentam compreender o discurso como quando tentam explicar ou predizer o comportamento do mundo físico. Segundo estas teoria, a capacidade para influenciar, controlar, iniciar ou predizer um fenômeno físico que está na base da sua compreensão deriva da construção de modelos de trabalho desse fenômeno. Frente a uma situação, tanto os elementos escolhidos para interpretá-la como as relações percebidas ou imaginadas entre eles determinam uma representação interna que é análogo-estrutural com a realidade percebida, de modo que atua como substituto dessa realidade. Da manipulação desses substitutos aparecem propriedades e relações não explícitas do sistema que podem ser 'lidas' de maneira direta, facilitando a realização de inferências e predições. Segundo Johnson-Laird (1983), os modelos mentais deveriam a sua origem à evolução da habili- dade de percepção dos organismos com sistema nervoso, sendo a percepção o que estabelece a mediação entre o mundo e o homem. Assim, os humanos (e possivelmente outros seres vivos) não apreendem o mundo diretamente senão através de uma representação interna dele, pois a percepção implica a construção de modelos mentais. Ou seja, a percepção de qualquer situação está condicionada pelos modelos mentais que somos capazes de construir. Além da percepção, os modelos mentais podem ser construídos a partir da experiência já internalizada e da interação com terceiros, como vimos no caso do discurso. Os modelos mentais gerados por estas fontes podem influenciar-se mutuamente; a percepção, por exemplo, pode ser influenciada pelos modelos gerados pela interação com terceiros (pense-se como se modifica nossa percepção dos ruídos noturnos depois da leitura de uma novela de terror) e vice-versa. Dentro da teoria postula-se que existem certos conceitos e procedimentos primitivos, i. é, inatos. Os demais conceitos devem ser construídos via modelos mentais, que deverão ser capazes de representar tanto a sua essência quanto a sua amplitude. O núcleo do modelo mental do conceito representará a essência deste, ou seja, as propriedades características do estado de coisas que o conceito descreve - por exemplo, o núcleo do modelo mental do conceito de telefone celular inclui a noção da comunicação sem fios - enquanto a amplitude do conceito estará determinada pelos procedimentos de manipulação dos modelos, de forma a definir o conjunto de estados de coisas possíveis que o conceito descreve - no exemplo anterior, telefone celular digital ou analógico. Este processo de construção dos conceitos via modelos mentais também é válido, segundo Johnson-Laird (ibid., p.415) para os conceitos abstratos. "Conceitos sem fundação [em modelos mentais] dificilmente são a base de todas as frases abstratas; no momento em que os indivíduos adquirem uma clara intuição acerca de como o mundo tem de ser para que uma afirmação seja verdadeira, devem ter transcendido uma representação meramente proposicional". Os núcleos direcionam a formação dos modelos mentais pois determinam a forma como o fenômeno é "visualizado". Tomemos um exemplo amplamente discutido como é o do conceito de força. Para os estudantes, uma característica essencial do conceito é a relação causal simples entre força e movimento, enquanto o essencial de tal conceito na visão da Física Clássica é a interação. Esses dois núcleos determinam formas diferentes de visualizar os fenômenos vinculados ao conceito de força, envolvendo a construção de modelos mentais "essencialmente" distintos em cada caso. Esta diferença na forma de visualização dos fenômenos seria um dos fatores para as dificuldades que os estudantes têm com os conceitos da Física Clássica. Apesar da importância desta questão, ela raramente é explicitada no ensino da Mecânica Clássica.

O caso da Mecânica Quântica não é diferente. Aliás, a dificuldade nesse caso é maior. Como indicamos 
antes, seus fundamentos determinam uma forma de percepção dos fenômenos microscópicos diferente da proporcionada pela Mecânica Clássica. Desde este referencial, então, as dificuldades que os estudantes universitários enfrentam para a compreensão da Mecânica Quântica derivam fundamentalmente da dificuldade de substituir os núcleos dos modelos mentais que servem para explicar o 'mundo clássico', impedindo-os de construir modelos mentais que lhes permitam visualizar "quanticamente" os fenômenos microscópicos. Nesses núcleos deveriam estar incorporados, entre outros, os conceitos de superposição de estados, dualidade ondapartícula e caráter probabilístico dos resultados de medidas.

A questão é, então, como fazer para que os alunos gerem modelos mentais adequados para a percepção quântica dos fenômenos microscópicos. Acreditamos que uma solução possível seja, sobretudo em cursos introdutórios, salientar diretamente as características quânticas dos sistemas ao invés de buscar analogias clássicas que reforçam as concepções clássicas dos estudantes, e usufruir das inúmeras experiências com uma ou poucas partículas, disponíveis hoje em dia, em geral conceitualmente simples, para que os estudantes possam familiarizar-se com a "percepção" quântica. De alguma forma, tais recentes experiências - muitas delas realizações físicas dos antigos "gedanken-experiments", outras visando derrubar mitos clássicos às vezes embutidos em paradoxos - propiciam a formação de uma nova geração de físicos que vem adquirindo uma compreensão natural, "intuitiva", dos fenômenos quânticos [13]. Ainda que não se pretenda que estudantes de outras carreiras possam atingir tal nível de compreensão, acreditamos que esta proposta [14] pode ser uma alternativa viável para um ensino mais eficiente dos conceitos básicos de Mecânica Quântica nos cursos introdutórios. A abordagem aqui proposta é entendida como fenomenológico-conceitual. Fenomenológica para propiciar a criação de uma nova percepção, e conceitual na medida em que os fenômenos escolhidos devem ser suficientemente simples (elementares) e dirigidos de forma a que a essência semântica dos (primeiros) conceitos envolvidos fique evidente.

\section{Discussão dos conceitos físicos fundamentais}

Dada a posição singular que a Mecânica Quântica ocupa dentro da Física - ainda que seu modelo matemático, seu formalismo abstrato, esteja firmemente estabelecido ${ }^{3}$, a interpretação da teoria continua, como indicamos na Introdução, ainda hoje, provocando acirrados debates -, consideramos importante esclarecer que neste trabalho adotamos a interpretação da Mecânica Quântica decorrente da formulação de Von Neumann. Diferentemente da interpretação de Copenhague, na interpretação ortodoxa os estados quânticos (representados pela função de onda) possuem uma realidade independente da medida ${ }^{4}{ }^{5}$. Embora o debate dessas questões e o confronto entre as distintas interpretações se convertam, sem dúvida, em uma experiência que permite apreciar profundamente as brechas entre os conceitos clássicos e quânticos, acreditamos, porém, que primeiro é indispensável que os estudantes aprendam os conceitos básicos em uma interpretação - e neste caso por que não na da interpretação ortodoxa, ou seja, aquela aceita usualmente nos meios acadêmicos [16]-, antes de poder se confrontar com outras interpretações. Sem o pré-requisito da compreensão de uma interpretação (a ortodoxa, por exemplo), outras interpretações ficam sem sustentação.

A estratégia didática pretende, então, tornar palpáveis alguns dos primeiros princípios que, em nosso entender, são fundamentais para compreender o mundo microscópico, devendo estar, portanto, nos núcleos dos modelos mentais necessários para a compreensão deste mundo. Apresentamos, a seguir, uma breve descrição da interpretação utilizada na proposta para os conceitos que consideramos centrais para esta proposta.

\section{Superposição de estados}

O princípio da superposição de estados é considerado por alguns pesquisadores como o postulado zero da Mecânica Quântica, sendo a superposição de estados um conceito fundamental tanto para entender-se o mundo microscópico, como também para ilustrar uma concepção que, ainda que com a mesma formulação matemática nas teorias quântica e clássica, implica em significações completamente diferentes.

Em linguagem vetorial - onde cada vetor representa

\footnotetext{
${ }^{3}$ Como bem observa Jammer [15], nenhum outro formalismo com uma estrutura drasticamente diferente daquela da Mecânica Quântica foi jamais aceito como alternativa.

${ }^{4}$ Embora não possuam, em geral, propriedades dinâmicas específicas.

${ }^{5}$ Freire Jr. (comunicação pessoal) ressalta, no entanto, que não é possível associar exclusivamente uma interpretação objetiva da Mecânica Quântica (atribuir um caráter objetivo ao conceito de estado de um sistema quântico, tornando-o menos dependente dos processos de medição) à interpretação decorrente da formulação de von Neumann. Segundo ele, a abordagem de von Neumann, tratando os aparelhos de medição como objetos quânticos, diluiu os limites entre o mundo cotidiano e o mundo quântico, abrindo a possibilidade de se cogitar de uma interação significativa entre a consciência do pesquisador e o sistema quântico, postura que inaugurou uma linha de abordagem subjetivista do problema da medida desenvolvida posteriormente por London, Bauer, von Weizscker e Wigner (embora o próprio von Neumann não tenha discutido o tema). Freire Jr. ressalta também que, em 1999, Michel Paty [17] propôs uma extensão do significado usualmente atribuído aos conceitos de "estado físico" e "quantidade física" de um sistema, de modo a permitir falar de "sistemas quânticos reais" como tendo "propriedades físicas" definidas, sem qualquer mudança teórica na Mecânica Quântica.
} 
um estado puro - este princípio pode ser expresso como segue.

Se os vetores $\mid \varphi_{1}>$ e $\mid \varphi_{2}>$ representam possíveis estados (puros) de um sistema, qualquer vetor $\left|\varphi_{3}\right\rangle=$ $c_{1}\left|\varphi_{1}>+c_{2}\right| \varphi_{2}>$ representa também um possível estado do sistema.

Tal princípio não pode formar parte de uma teoria determinista, pois nestas teorias, ainda que qualquer vetor $|\varphi\rangle$ possa ser escrito como um somatório de vetores $\left|\varphi_{i}\right\rangle$, não podemos considerar qualquer vetor construído desta forma como representando um possível estado (puro) com realidade física. Ou seja, restringindo-nos ao caso bidimensional, onde existem dois possíveis resultados $x_{1}$ e $x_{2}$ para uma dada medida, representados pelos subespaços $E_{1}$ e $E_{2}$, os vetores (normalizados) $\mid \varphi_{1}>$ e $\left|\varphi_{2}\right\rangle$, pertencentes aos subespaços $E_{1}$ e $E_{2}$, respectivamente, podem representar estados puros, enquanto o vetor $\left|\varphi_{3}\right\rangle=\left(\frac{1}{2}\right)\left|\varphi_{1}\right\rangle$ $+\left(\frac{1}{2}\right)^{1 / 2}\left|\varphi_{2}\right\rangle$, em uma teoria determinista, não pode. Por isto, Dirac [19] afirma que a maior diferença entre a Mecânica Clássica e a Mecânica Quântica decorre do princípio de superposição de estados.

Portanto, uma teoria que inclua este princípio deve ser inerentemente probabilística: as probabilidades de resultados de um determinado experimento podem ser diferentes de 0 ou 1 [20:108]. Rompe-se, desta forma, a identidade que a Física Clássica estabelece entre estado de um sistema e resultado de uma medição, ou seja, o resultado de uma única medição não nos pode informar, em geral, de forma completa acerca do estado do sistema antes da medida, ocorrendo apenas relações em forma probabilística.

Os questionamentos à "realidade física" deste princípio foram formulados pelo próprio Schrödinger, na forma do famoso paradoxo do gato, sendo muitas as vozes a dizer que a "superposição de estados" é só um efeito matemático decorrente do desconhecimento sobre o estado do sistema. Este questionamento, porém, tem se mostrado incorreto, havendo-se conseguido verificar a superposição de estados em um único sistema" em pequena escala ${ }^{6}$ [21]. Por outra parte, desenvolvimentos teóricos recentes [22] permitiram tornar mais claro o porquê de não serem observados efeitos de superposição na escala macroscópica: basicamente, porque os sistemas macroscópicos não são sistemas fechados, mas sim que interagem dissipativamente com o meio.

O princípio da superposição de estados é trabalhado sem grandes dificuldades matemáticas pelos alunos em cursos tradicionais de Mecânica Quântica, quando se analisam estados de norma finita. Contudo, para a maioria dos estudantes ele se torna apenas uma decomposição matemática, sem uma contrapartida na realidade física, identificando-se com a concepção clássica [8]. Dificilmente os estudantes se deparam, em cursos introdutórios (e até em alguns mais avançados), com as nuances deste princípio e suas conseqüências físicas. Mais ainda, apesar da sua importância, raramente é discutido nos cursos introdutórios de Física Geral e também raramente aparece nos livros utilizados nestas disciplinas.

\section{Dualidade onda-partícula e Princípio de Incerteza}

Assim como para Dirac o princípio de superposição linear de estados é o postulado mais importante da Mecânica Quântica, para outros a dualidade ondapartícula ou sua versão formalizada no princípio de incerteza são as inovações cruciais da Mecânica Quântica. Neste contexto diz-se que, no mundo microscópico, os aspectos corpusculares e ondulatórios da matéria são complementares, ou seja, um sistema quântico pode exibir aspectos corpusculares ou aspectos ondulatórios dependendo do arranjo experimental, mas não ambos ao mesmo tempo ${ }^{7}$. Em decorrência, não é possível montar um dispositivo experimental onde os dois aspectos possam ser revelados ao mesmo tempo.

Este aspecto complementar do mundo microscópico é expresso, no formalismo da Mecânica Quântica, nas relações de incerteza. Segundo o Princípio de Incerteza, enunciado por Heisenberg em 1927, dado um par de grandezas físicas $A$ e $B$, representadas pelos operadores A e B que não comutam, quanto maior é o conhecimento de uma delas em um dado experimento, menor é o conhecimento da outra. Matematicamente,

Se $[A, B] \neq 0$, então, $\Delta A \cdot \Delta B \leq \frac{\hbar}{2}$. sendo $\Delta A$ a incerteza no operador $A$ e $\Delta B$ no operador $B$.

Este princípio refere-se, então, aos tipos de observáveis que podem ser encontrados na teoria e pode ser lido desde duas perspectivas [25:cap.1]. Por uma parte podemos considerá-lo como uma propriedade fundamental do estado físico de um sistema quântico de forma que, independentemente de como seja preparado tal estado, não pode corresponder a valores precisos de dois observáveis incompatíveis; por exemplo, não pode ter valores precisamente definidos de posição e momento. No mesmo sentido, um pacote de ondas clássico não pode ter valores perfeitamente definidos de posição e número de onda. A outra leitura (que

\footnotetext{
${ }^{6}$ No paradoxo original, os estados do gato estavam "emaranhados" (ver seguinte nota de rodapé) com os estados possíveis do átomo. Os estados que na literatura se conhecem como "estados do gato de Schrödinger", na verdade, não são estados emaranhados e, sim, superposições coerentes de estados distinguíveis de um sistema único [23].

${ }^{7}$ Pessoa Jr. [24] chama a este enunciado da dualidade onda-partícula de "versão forte" (basicamente defendido por Niels Bohr). Segundo Pessoa Jr. (p. 29), o princípio pode ser enunciado de forma mais fraca, a saber: "Qualquer radiação ondulatória é detectada em pacotes mínimos de energia ou massa e qualquer partícula individual pode exibir fenômenos tipicamente ondulatórios, como a interferência".
} 
pode ser pensada a partir do "microscópio de Heisenberg") é considerar o Princípio de Incerteza como uma propriedade fundamental do processo de medida, de modo que a incerteza, por exemplo, no momento é devida à perturbação do sistema no processo de medida. Ambas as leituras têm, no entanto, uma raiz comum: se o dispositivo de medida, segundo a primeira leitura, apresenta incertezas inevitáveis, necessariamente a medição de uma grandeza perturbará a outra; por outra parte, as perturbações que inevitavelmente acompanham a medição de grandezas incompatíveis nos impedirão de preparar um sistema quântico em um estado com valores simultâneos bem definidos de grandezas incompatíveis. De qualquer forma, o Princípio de Incerteza é uma limitação inerente a todo ato de medição e não pode ser superado com os avanços tecnológicos dos sistemas de medição. Este limite natural à nossa capacidade de observação leva-nos a abandonar idéias centrais da visão clássica de mundo, como a do caráter fundamental que o conceito de trajetória ocupa aí .

Assim como no caso do princípio de superposição, toda teoria que contenha um princípio sobre observáveis incompatíveis é inerentemente probabilística, ou seja, permite que as probabilidades de resultados de um determinado experimento sejam diferentes de 0 ou 1. Somente se todos os observáveis de uma teoria forem compatíveis (como é o caso da Física Clássica), a teoria pode ser determinística.

Ainda que, tanto o princípio de superposição de estados quanto o de incerteza, impliquem em teorias probabilísticas, os dois são conceitualmente diferentes, ou seja, que uma teoria tenha observáveis incompatíveis não implica que estados puros possam ser somados e vice-versa ${ }^{8}$.

O Princípio de Incerteza nos permite estabelecer, pela primeira vez na história da Ciência, uma escala "absoluta" de tamanho: o mundo atômico e subatômico é pequeno no sentido absoluto, porque qualquer medição efetuada nessa escala usará recursos da mesma escala e poderá afetar o resultado, de forma que é preciso sempre indicar como estão sendo observados os fenômenos. Ou seja, na escala atômica e subatômica existem limitações absolutas às possibilidades de medição, questão esta enfatizada por Dirac (1930)[19].

Os estudantes, porém, são apresentados, em geral, somente às fórmulas vinculadas ao Princípio de Incerteza, sem que sejam estabelecidas, pelo menos nos cursos introdutórios, as suas relações com o conceito de dualidade ou com a idéia de quantização. Assim, muitos dos estudantes aprendem as fórmulas sem saber onde ou como aplicá-las. E, apesar de aceitar-se que as relações de incerteza não emergem de imperfeições tecnologica- mente superáveis nos dispositivos de medição, esta é a interpretação mais difundida possivelmente porque este é o contexto em que os estudantes de ciências primeiramente se deparam com a palavra "incerteza" 9 [6],[8].

\section{Caráter probabilístico dos resultados de medidas e problema da medida}

A Mecânica Quântica, como decorre dos princípios discutidos anteriormente, é uma teoria inerentemente probabilística: enquanto na Mecânica Clássica o resultado de cada medição pode ser previsto com precisão arbitrária, desde que o estado inicial seja conhecido, a Mecânica Quântica, nas mesmas condições, oferece só predições probabilísticas. A natureza dessas probabilidades, por outra parte, difere das da Física Clássica: elas não acontecem devido à falta de conhecimento, pois a função de onda contém toda a informação acerca do estado de um sistema, e as densidades de probabilidades apresentam termos de interferência porque são resultantes do módulo ao quadrado de somas de amplitudes. Além disso, em contraposição à Física Clássica, a relação entre o sistema a ser medido e o dispositivo de medida é simétrica e biunívoca [26]: assim como o sistema deve modificar o dispositivo de medida (para mover o ponteiro, por exemplo), o dispositivo modifica o estado do sistema que fica, em geral, depois de completada a medida, em um estado diferente. O que acontece com o sistema durante a medida? Como deve ser o dispositivo de medida para realizar as medições desejadas? Essas questões, que não aparecem no caso clássico, constituem o que se denomina de problema da medida em Mecânica Quântica.

A teoria da medida em Mecânica Quântica foi assunto central nos debates das décadas de vinte e trinta, quando se desenvolvia a compreensão a respeito das medições para um único objeto. Nas décadas seguintes, porém, esse interesse passou, pois os sistemas com os quais os físicos trabalhavam, ainda que mecanoquânticos em princípio, só faziam uso da interpretação probabilística do quadrado das amplitudes da função de onda. Segundo Thorne [25:2], "não havia necessidade de invocar-se o que rapidamente foi visto como o esotérico, problemático e duvidoso 'colapso (redução) da função de onda'." A questão foi retomada com mais força na década de oitenta, quando os desenvolvimentos tecnológicos começaram a permitir a realização de experimentos de medições repetidas sobre um único sistema $^{10}$

O que ocorre com o sistema durante uma medição não pode ser deduzido dos princípios anteriores, nem da equação de Schrödinger, que governa o comportamento dos sistemas quânticos. A equação de Schrödinger é

\footnotetext{
${ }^{8}$ Porém, como indica Hughes [20], a existência de observáveis incompatíveis permite distinguir entre estados puros e mistos.

${ }^{9}$ As idéias de "erro na medição", e de "medidas incorretas" são as que aparecem mais vezes associadas à de incerteza, nos pré-testes usados em nossa pesquisa.

${ }^{10}$ Isto não quer dizer que a questão da medida não tivesse importantes desenvolvimentos em períodos anteriores [27].
} 
uma equação de evolução temporal determinista (o estado final é determinado univocamente pelo estado inicial) e reversível (do estado final pode-se em princípio voltar ao estado inicial), não podendo governar ou descrever um processo de medida inerentemente probabilístico. Tal processo é resgatado pelo postulado da filtragem da função de onda, que incorpora a irreversibilidade nas medidas (depois de efetuada a medição não é possível voltar ao estado anterior à medida) e o não-determinismo (antes da medição não se pode predizer em que estado se encontrará o sistema depois da medição) $)^{11}$.

O problema da medida é uma questão praticamente omitida nos cursos tradicionais de Mecânica Quântica (os cursos de introdução não tratam sobre o tema), em parte devido à sua complexidade e em parte porque muitos dos livros utilizados nestas disciplinas foram escritos nas décadas em que este problema não era um tema de pesquisa relevante. Porém seu tratamento é importante, pois encerra, em si mesmo, as diferenças fundamentais do tratamento quântico. Como discutido em Greca e Moreira (1999) [8], estudantes de cursos avançados de graduação parecem manter idéias clássicas a este respeito.

\section{Descrição da implementação da proposta}

Implementamos, sob a perspectiva mencionada, no primeiro semestre de 1999, uma experiência-piloto ${ }^{12}$ em uma turma da disciplina de Física Geral do quarto semestre dos cursos de Engenharia da UFRGS, constante de 25 estudantes, com a concordância e colaboração da professora regular da turma. O projeto, desenvolvido durante 24 horas- aula, incluiu os seguintes temas: computação quântica, experiência de interferência para sistemas quânticos, princípio de incerteza, experiência de Stern-Gerlach e sistemas de dois estados, superposição linear de estados, resultados de medições, distribuição de probabilidades, equação de Schrödinger, problemas simples de autovalores (poços e barreiras de potencial), tunelamento e microscópio eletrônico de varredura, átomo de hidrogênio, espectros e saltos quânticos, paradoxo do gato de Schrödinger, medições sem interação com o objeto, laser e confinamento de objetos quânticos com técnicas de resfriamento. Em todas as aulas, a preocupação dominante foi a de apresentar os fenômenos, destacando-se os conceitos envolvidos e incentivando-se os estudantes, através das questões propostas e das discussões, a (re)criar a sua percepção. As discussões em grupos e com a professora foram, portanto, parte fundamental do processo. Cabe notar que, depois da terceira aula, todos os conceitos fundamentais selecionados por nós (estado - função de onda, princípio de incerteza, superposição linear de estados, resultados de medições, distribuição de probabilidades) já haviam sido apresentados aos alunos, sendo em cada nova aula retomados na "leitura" dos novos fenômenos, buscandose assim a diferenciação conceitual progressiva ${ }^{13}$.

Cabe ressaltar que esses foram os únicos conteúdos tratados: as experiências que tradicionalmente são apresentadas aos estudantes - como radiação do corpo negro ou efeito Compton, não foram incluídas. Estas experiências formam parte da estratégia tradicional para o ensino introdutório da Mecânica Quântica, que, como indicado na Introdução, salienta aspectos históricos que apelam mais para às características clássicas dos sistemas do que para às quânticas e cujos resultados, em termos dos aprendizagens alcançados pelos estudantes, não são satisfatórios.

Dado que o formalismo matemático é inerente à descrição quântica (como também, aliás, à clássica), uma das preocupações no projeto foi introduzi-lo em simultaneidade com os conceitos físicos. Para isso, trabalhamos sobre a base de sistemas de dois estados (em particular, o de spin do elétron), que consideramos seria de melhor compreensão para os estudantes, para discutir as questões da superposição linear de estados, distribuição de probabilidades e a diferença entre estado de um sistema e resultado de uma medição. Acreditamos que pensar em termos de vetores de espaços bidimensionais permite aos estudantes pensar de forma mais concreta, a perceber melhor o que está envolvido nos princípios fundamentais, e que pode ficar mascarado quando esses são apresentados de forma mais geral. Ou seja, tais sistemas podem ser imaginados de forma mais simples, facilitando assim a formação de modelos mentais. O uso de sistemas de dois estados não é, de modo algum, uma inovação pois aparece em vários textos clássicos sobre Mecânica Quântica (por exemplo, [31-33]). Contudo, como o tema do spin quase não é tratado nos cursos introdutórios, esses sistemas, muitas vezes, não são abordados. Como os alunos apresentavam pouca familiaridade com o trato de equações diferenciais, depois de introduzir o conceito de (equação de) autovalores e de discutir a questão da quantização da energia, lançamos mão de recursos computacionais

\footnotetext{
${ }^{11} \mathrm{O}$ único tipo de medição que não cumpre com estas condições é o denominado "medida quântica perfeita não demolidora" em que o sistema já se encontra em um autoestado do observável a ser medido [24].

${ }^{12}$ Esta proposta foi utilizada em duas outras turmas da mesma disciplina no segundo semestre de 1999.

${ }^{13} \mathrm{~A}$ diferenciação progressiva é um princípio, proposto por David Ausubel [28], [29], que se aplica tanto à dinâmica da estrutura cognitiva como à programação do conteúdo. Do ponto de vista cognitivo, significa que, à medida que ocorre a interação entre o conhecimento prévio e o novo conhecimento, as idéias- âncora vão adquirindo novos significados, maior clareza e estabilidade e, assim, maior capacidade de servir de ancoradouro para novos conhecimentos. Da ótica instrucional, sugere que as idéias mais gerais e mais inclusivas da matéria de ensino devem ser apresentadas no início do ensino para, somente então, serem progressivamente diferenciadas em seus pormenores e em suas especificidades [30].
} 
para mostrar como a quantização está regulada pelas soluções fisicamente aceitáveis para a função de onda associada a determinados sistemas. Outra importante ênfase do curso foi a de vincular o modelo físico a aplicações tecnológicas bastante recentes. A metodologia de sala de aula foi a de trabalho em pequenos grupos (cada grupo sendo formado por três ou quatro alunos), a partir de textos especialmente elaborados que continham, ademais, uma relação de questões conceituais e de pequenos problemas com os quais os alunos deviam trabalhar em aula, com o auxílio da professora. No Apêndice mostramos um destes textos.

\section{Metodologia de pesquisa}

Descrevemos, a seguir, os resultados da primeira implementação da proposta (estudo-piloto) e os comparamos com os de uma turma semelhante usada para controle. A turma de controle recebeu instrução em conteúdos introdutórios de Mecânica Quântica durante o mesmo período de tempo, mas com o enfoque tradicional e utilizando a literatura corrente na Física Geral (Física $I V$ de D. Halliday e R. Resnick e Física $I V$ de $\mathrm{P}$. Tipler). Os resultados também são comparados com os de um grupo de estudantes da disciplina do curso de Física de Introdução à Mecânica Quântica (IMQ), do curso de Física da UFRGS, que recebeu instrução em conteúdos introdutórios com uma abordagem semelhante à da turma de controle (abordagem tradicional), porém durante um período de tempo mais prolongado (um semestre, literatura básica: Fundamentos de Fúsica Moderna, de R. Eisberg). Interessava nos comparar o desempenho dos estudantes do grupo experimental com os do outro grupo, que, por um período bastante maior, recebeu instrução nas idéias fundamentais da Mecânica Quântica.

Dos vários instrumentos (testes de associação de conceitos, respostas complementares dos testes de associação de conceitos, questões conceituais, entrevistas, documentos escritos dos estudantes, questionário de avaliação da unidade por parte dos estudantes) - e técnicas de análise que utilizamos (análises interpretativa, de agrupamentos hierárquicos, multidimensional e multivariada) [34]; [35], apresentamos neste trabalho apenas os resultados decorrentes de um teste de associação de conceitos, examinando mediante a análise de agrupamentos hierárquicos [36].

A utilização dos testes de associação de conceitos, em que os sujeitos devem escrever logo abaixo de cada um dos conceitos selecionados para a pesquisa - apresentados ao aluno aleatoriamente, cada um no topo de uma folha em branco - tantos conceitos (ou palavras) quantos possam associar ao conceito apresentado, decorre do pressuposto de que entender como determinados conceitos estão associados pode dar indícios dos modelos mentais que permitiram percebê-los como semelhantes [37]. Ou seja, se os núcleos dos modelos mentais determinam como os conceitos ou os fenômenos são percebidos, deverão determinar também se certos conceitos e fenômenos são percebidos como semelhantes ou não.

Tradicionalmente, os conceitos escolhidos para construir um teste de associação de conceitos são os supostamente mais significativos da área que se pretende estudar, tanto a partir do número de vezes que aparecem em livros de texto como pelos critérios dos especialistas. No caso em foco, levamos essas duas razões em consideração, além de certas premissas decorrentes do referencial teórico. Assim, o conjunto de conceitos- chave devia conter alguns dos conceitos que consideramos fundamentais para entender o mundo quântico, bem como conceitos que se relacionassem mais à fenomenologia, de forma a observar se os estudantes vinculavam estes últimos aos conceitos considerados fundamentais (ou seja, para ver como os estudantes "visualizavam" os fenômenos quânticos). Foram assim elaboradas listas de conceitos com estas características e o conjunto final surgiu da discussão com um especialista. Acreditamos que esta forma de construção do teste lhe confere validade de conteúdo. Os conceitos que foram apresentados aleatoriamente aos estudantes foram: elétron, estado de um sistema físico, superposição linear de estados, função de onda, dualidade onda-partícula, princípio de incerteza, observável, observáveis simultâneos, autovalores, resultados de medida, probabilidade, trajetória, tunelamento, efeito fotoelétrico e valor médio.

Os dados fornecidos pelo teste de associação de conceitos foram transformados em um coeficiente de relacionamento (CR) entre pares de conceitos-chave, a través do método de Garskof \& Houston [38], já utilizado em outras pesquisas na área de ensino de ciências [39]; [40].

Este coeficiente varia de 0 a 1 e dá a medida da proximidade entre dois conceitos. Quanto maior é o valor de CR, maior é o relacionamento entre os conceitos. Os coeficientes de relacionamento são calculados para todos os pares de conceitos-chave e colocados sob forma matricial. Com base nas matrizes individuais para cada estudante são calculadas as matrizes médias dos coeficientes de relacionamento para grupos de estudantes. Esta matriz média constitui a matriz de similaridade que depois é submetida à Análise de Agrupamentos Hierárquicos.

Em relação à técnica, a Análise de Agrupamentos Hierárquicos (AAH) [36];[41], é utilizada para descobrir a existência de estruturas inerentes ao conjunto de medidas de similaridade. Essa técnica estabelece uma escala hierárquica entre os conceitos, com base em valores de proximidade (os coeficientes de relacionamento, por exemplo), de forma que, quanto mais afastado está o ponto de ligação (ou seja, quanto mais posterior for o estágio em que os conceitos são agrupados), menor é a interação entre eles. A operação básica realizada é a 
construção de agrupamentos por meio da combinação de dois ou mais conceitos por vez, começando-se num estágio em que todos os casos são considerados como agrupamentos separados, i. é, considerando-se inicialmente tantos agrupamentos como casos. No segundo estágio, dois ou mais dos casos são combinados em um único agrupamento. No terceiro estágio, tanto um terceiro caso pode ser incluído em um agrupamento já existente, como outros dois casos podem emergir em um novo agrupamento. Este esquema prossegue até o estágio em que todos os casos estão combinados em um único grupo. Uma vez que um agrupamento é formado, não pode mais dividir-se, podendo apenas se combinar com outros agrupamentos.

A escala resultante deste processo é apresentada em um diagrama denominado "dendograma". Nos resultados apresentados a seguir, a distância entre os conceitos está re-escalada para um intervalo de 0 a 25 . Como o valor 25 pode representar uma distância infinita entre os conceitos, ou seja, que não tenham qualquer interação (e portanto, os agrupamentos feitos nesse ponto não serem significativos), foi tomada como valor de corte para a análise dos agrupamentos significativos a distância 20. A essa distância acontecem as maiores interações entre os conceitos para os AAH de dois especialistas que responderam ao teste.

\section{Resultados da análise de agrupamentos hierárquicos}

Na Tabela 1 aparecem os valores de CR (coeficiente de relacionamento médio) para cada grupo. O aumento no valor de CR no pós-teste, comparado com o valor antes da instrução, foi estatisticamente significativo para os três grupos. A análise multivariada, comparando-se os valores médios de $\mathrm{CR}$ antes da instrução para cada grupo mostra que não existem diferenças significativas entre os grupos, ao nível $<0,001$. Este resultado era esperado, pois é a primeira vez que todos esses estudantes cursam alguma disciplina com conteúdos de Mecânica Quântica. Na terceira fila da tabela, aparece a estimativa do efeito do tratamento para cada grupo, tal como medido pelo índice. Esse índice é o quadrado da razão de correlação e indica quanto da variância dos escores individuais é atribuída aos grupos.

\begin{tabular}{|c|c|c|c|}
\hline Grupo & $\begin{array}{c}\text { Física Geral IV } \\
\text { Experimental }\end{array}$ & $\begin{array}{c}\text { Física Geral IV } \\
\text { Controle }\end{array}$ & $\begin{array}{c}\text { Introdução } \\
\text { Mecânica Quântica }\end{array}$ \\
\hline Pré Teste & 0,14 & 0,13 & 0,14 \\
Pós-teste & 0,20 & $0,14 \diamond$ & $0,17^{\star}$ \\
$\eta^{2}$ & 0,48 & 0,07 & 0,27 \\
\hline
\end{tabular}

Tabela 1: Valores de CR (médio) para os grupos estudados

- Incremento estatisticamente significativo $(<0,001)$.

- Incremento estatisticamente significativo $(<0,005)$

Da tabela se depreende que, após a instrução, os estudantes dos três grupos considerados estabelecem mais relações entre os conceitos escolhidos. Porém, para os estudantes do grupo experimental, as diferenças entre o pré e pós-teste são maiores.

Na Fig. 1 aparece o dendograma do pré-teste correspondente ao grupo experimental e nas Figs. 2, 3 e 4 os dendogramas dos pós-testes correspondentes ao grupo experimental (considerando-se somente os estudantes que também responderam o pré-teste, $\mathrm{N}=16$ ), ao grupo de controle $(\mathrm{N}=10)$ e ao grupo IMQ $(\mathrm{N}=11)$, respectivamente. As interpretações dos dendogramas foram consensuadas entre os três pesquisadores.

Vários dos agrupamentos que aparecem na Fig. 1, que poderíamos pensar serem significativos, parecem decorrer de uma associação mecânica entre palavras semelhantes, efeito que pudemos observar nas listas de palavras escritas pelos sujeitos. Isso acontece nos agrupamentos de elétron com efeito fotoelétrico (palavra comum elétron), observáveis - observáveis simultâneos (palavra comum observáveis) e dualidade onda-par

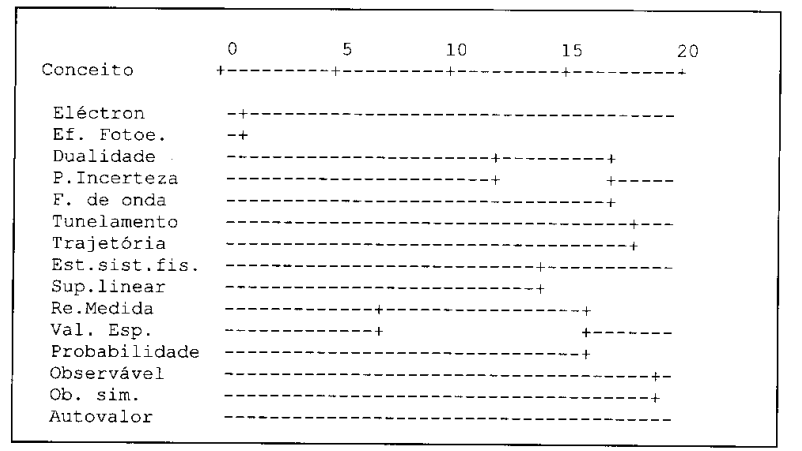

Figura 1. Agrupamentos hierárquicos do pré-teste - Alunos do grupo experimental.

tícula - função de onda (palavra comum onda). Os outros dois agrupamentos também são exemplos de associações estabelecidas entre os conceitos em outros contextos. O agrupamento entre estado de um sistema físico e superposição linear de estados é feito pelos estudantes a partir da Termodinâmica, i. é, eles associam, com estado, os estados de agregação da matéria (sólido, liquido, gasoso) e associam, com superposição, 
a coexistência desses estados (colocando sólido-líquido, sólido-gasoso etc). Isso é de esperar, sendo no contexto da Termodinâmica em que os estudantes trataram fundamentalmente do conceito de estado. O agrupamento entre trajetória e tunelamento decorre, provavelmente, da associação estabelecida no âmbito do cotidiano entre caminho e túnel. O agrupamento mais interessante, no contexto de expressar idéias físicas que podem confrontar-se com as que esses estudantes encontraram nas aulas de Mecânica Quântica, é o formado por resultado de uma medida - valor esperado - probabilidade. Esse agrupamento, possivelmente, reflete uma idéia clássica associada à probabilidade: se os resultados de uma medição efetuada sobre um sistema não coincidem com os valores esperados calculados, é pela presença de erros na medição. Esses resultados devem então ser tratados estatisticamente.

De fato, a instrução dada aos alunos foi a de que associassem com aquilo que lhes viesse à cabeça. Portanto, é de esperar que as associações observadas nos pré-testes desses grupos sejam menos significativas, mais mecânicas, e vinculadas, em muitos casos, pela similaridade morfológicas dos conceitos envolvidos. Isso difere do que acontece nos pós-testes, em que os estudantes estão motivados a circunscrever-se ao âmbito do tema pesquisado, e em que, por terem visto alguns dos conceitos durante as aulas, formaram alguma idéia a respeito deles, tendendo a explicar o que entenderam.

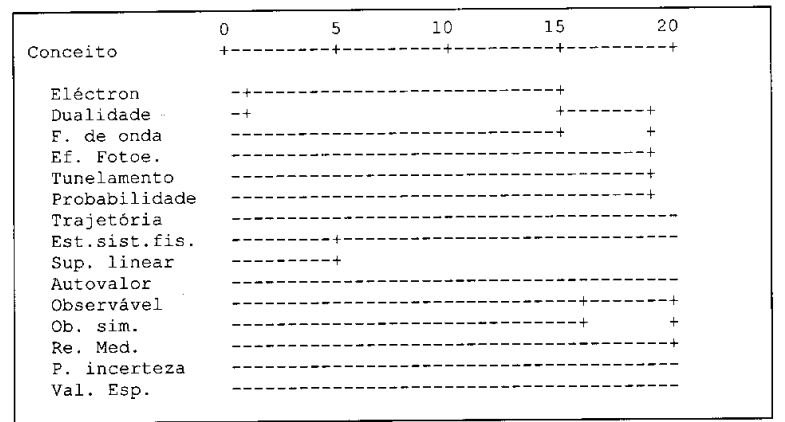

Figura 2. Agrupamentos hierárquicos do pós-teste - Alunos do grupo experimental.

Pode-se ver, na Fig. 2 correspondente ao pós-teste do grupo experimental, que os dois elementos mais associados são elétron e dualidade, associação que pode ser explicada como decorrência de os sujeitos entenderem o elétron como uma partícula quântica com comportamento dual. Junta-se a este agrupamento o conceito de função de onda, e depois os conceitos de efeito fotoelétrico, tunelamento e probabilidade. Esse agrupamento é significativo: nele aparecem elementos fenomenológicos (elétron, tunelamento e efeito fotoelétrico) junto a conceitos quânticos que servem para descrevê- los. Poderíamos sintetizar este agrupamento na seguinte frase, montada levando em conta a ordem da associação: "O elétron é uma partícula quântica com comportamento dual, expresso pela função de onda, comportamento este que permite explicar o efeito fotoelétrico e o tunelamento, por meio da determinação da probabilidade". Este agrupamento reflete uma característica apontada na análise qualitativa [42], qual seja a de os fenômenos serem explicados a partir de conceitos (mais fundamentais).

Aparece, a seguir, um agrupamento entre estado de um sistema físico e superposição de estados. Essa associação difere, na nossa concepção, da associação semelhante surgida no pré-teste. Os estudantes não mais associariam com estado de um sistema físico conceitos derivados da Termodinâmica, senão que agora os conceitos estariam associados como conseqüência de que o estado de um sistema físico pode ser uma superposição de estados de um dado observável. Por último, aparecem agrupados observáveis e observáveis simultâneos. Note-se que tal agrupamento se junta ao de resultado de uma medição. Diferentemente do préteste, em que o valor esperado estava agrupado com o resultado de uma medida (representativo de uma relação clássica), tal agrupamento se desfaz e o resultado de uma medida se associa ao agrupamento de observáveis. Possivelmente, isso seja reflexo do fato de os estudantes estarem entendendo que o resultado da uma medida depende das grandezas medidas. Aparecem, sem agrupar-se, os conceitos de trajetória, autovalor, Princípio de Incerteza e valor esperado. A não-associação, com outros, do Princípio de Incerteza é reflexo da escassa importância dada a esse princípio por um numero significativo de estudantes, tal como visto na análise qualitativa complementar, não incluída neste trabalho. O primeiro agrupamento, então, descreve fenômenos físicos; o segundo descreve o estado do sistema, e o terceiro se refere ao que acontece depois da medida. Em resumo, ainda que algumas associações desejáveis estejam ausentes, as associações estabelecidas por estes estudantes são todas corretas e os agrupamentos são mais significativos que os do pré-teste.

Diferentemente do caso anterior, a Fig. 3 correspondente ao pós-teste dos estudantes do grupo de controle mostra um escasso relacionamento entre os conceitos: dos quinze conceitos arrolados, sete não se relacionam com os restantes. O primeiro agrupamento que aparece é o de elétron e probabilidade, juntando-se depois efeito fotoelétrico e tunelamento. Esse agrupamento reúne conceitos fenomenológicos, aos quais se adiciona, porém, somente o conceito fundamental de probabilidade. Isso pode significar que os fenômenos não sejam compreendidos a partir de conceitos mais fundamentais. Aparece depois um agrupamento entre função de onda e dualidade e, por último, entre resultado de medida e valor esperado, o que, como indicado anteriormente, reflete uma associação clássica. Ou seja, neste caso, além de existirem poucos agrupamentos, alguns deles são pouco significativos ou incorretos do ponto de vista da Mecânica Quântica. 


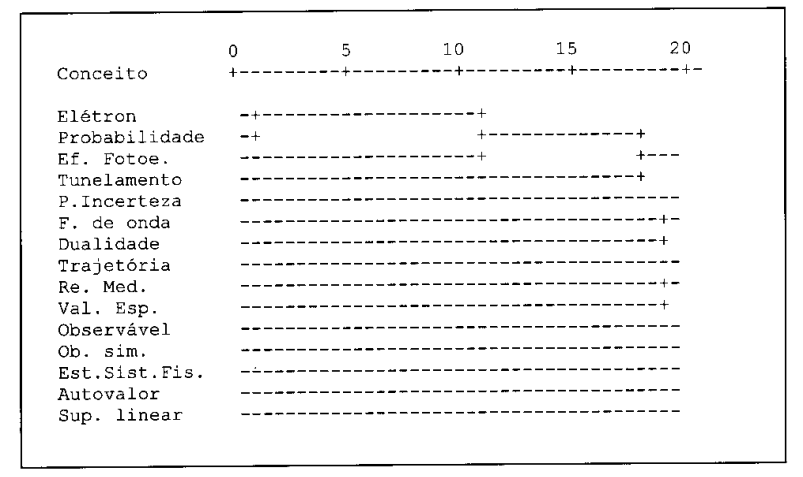

Figura 3. Agrupamentos hierárquicos do pós-teste - Alunos do grupo de controle.

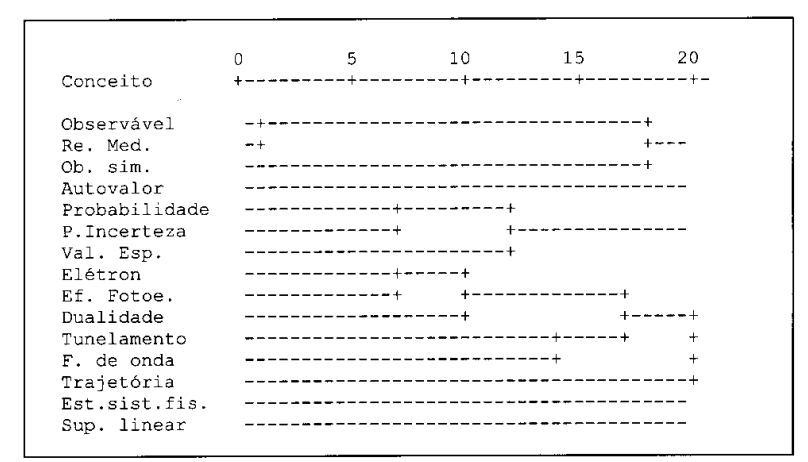

Figura 4. Agrupamentos hierárquicos do pós-teste - Alunos do grupo IMQ.

Na Fig. 4, que corresponde ao pós-teste dos estudantes da IMQ, o primeiro agrupamento que aparece é o de observável com resultado de medida e com observável simultâneo, semelhante ao terceiro agrupamento do grupo experimental. Aparece depois um agrupamento entre probabilidade, Princípio de Incerteza e valor esperado, agrupamento que possivelmente refletia a idéia de que, se não é possível determinar com certeza o valor de uma grandeza física, só podemos conhecer o seu valor provável (valor esperado) e que este é afetado pela probabilidade de ocorrência dos diferentes possíveis resultados. Aparece depois um grande agrupamento, onde conceitos mais fenomenológicos como o de elétron, efeito fotoelétrico, tunelamento e trajetória aparecem junto a conceitos mais fundamentais como função de onda e dualidade. Porém, diversamente do agrupamento semelhante no grupo experimental, aqui aparece também o conceito de trajetória, em princípio um conceito clássico. Uma leitura, neste caso, poderia ser: "O elétron é uma partícula que apresenta efeito fotoelétrico e isso evidencia seu comportamento dual. A função de onda dessa partícula dá conta do tunelamento, trajetória percorrida pela partícula". Finalmente, os conceitos de superposição de estados e de estado de um sistema físico não aparecem relacionados nem entre si nem com qualquer outro. Neste caso, então, os dois primeiros agrupamentos podem ser pensados como representando elementos após a medição, enquanto o terceiro descreve fenômenos (incluindo-se entre eles o de trajetória). Sob essa perspectiva, diríamos que a estes estudantes está faltando conceber como é o sistema antes da medida. Este grupo apresenta alguns agrupamentos semelhantes aos do grupo experimental, porém as associações estabelecidas parecem ser menos significativas e, em um caso, incorreta.

Os resultados desta análise, no que concerne ao grupo experimental, mostram que, comparativamente com os outros dois grupos, estabeleceu mais relações entre os conceitos, mostrando, parece, que a aprendizagem dos conceitos, na medida em que pode ser evidenciada por esta técnica (número e qualidade das associações), foi mais significativa nos estudantes que receberam instrução com a abordagem proposta do que nos estudantes dos outros dois grupos, que receberam instrução com a abordagem tradicional.

\section{Considerações finais}

Os resultados obtidos com outras turmas em outra oportunidade, assim como com outros instrumentos e técnicas de análises [14], [34], [42] parecem também indicar que a abordagem fenomenológico-conceitual utilizada foi bem sucedida no que diz respeito à incorporação, por parte de um número significativo dos estudantes, de conceitos fundamentais para a compreensão de fenômenos microscópicos. Estes estudantes conseguem fazer predições corretas e dar explicações consideradas adequadas para este nível de aprendizagem a respeito de vários destes fenômenos mostrando, ao que parece, a formação de modelos mentais com núcleos consistentes com a fenomenologia quântica. Isso, aparentemente, não é conseguido na abordagem tradicional não só durante período semelhante de tempo senão, também, durante um período bem maior.

Além disso, a utilização de resultados atuais de pesquisa e de inovações tecnológicas não só foi importante para o processo de formação de uma nova forma de percepção dos fenômenos microscópicos como também motivou os estudantes, muitos dos quais passaram a considerar a Mecânica Quântica como uma porta a novos horizontes, inclusive no seu campo profissional. É interessante destacar que o clima de discussão, envolvendo a grande maioria dos estudantes, caracterizou as aulas. Os alunos se sentiram exigidos a compreender fenômenos desde uma perspectiva em confronto com a Mecânica Clássica e, ainda, que nem todos tenham conseguido fazê-lo, todos parecem ter percebido que a Mecânica Quântica fornece um novo olhar sobre os fenômenos microscópicos. A professora regular da turma, que já ministrou cursos similares em várias oportunidades, ficou agradavelmente surpresa pelo envolvimento demonstrado pelos estudantes. Esse interesse parece haver-se mantido para a área seguinte do 
curso - noções de Estado Sólido. Por último, 84\% dos estudantes avaliaram positivamente o projeto, sugerindo que os alunos, embora bastante exigidos durante as aulas, as vivenciaram como uma boa experiência de aprendizagem. Embora nossa proposta tenha sido implementada em turmas das carreiras de Engenharia, cremos que a necessidade de uma mudança no ensino introdutório de conteúdos quânticos também é de fundamental importância para os estudantes dos cursos de Licenciatura. Hoje é relativamente fácil encontrar muitos dos novos fenômenos e aplicações decorrentes dos fundamentos da Mecânica Quântica em revistas e livros de divulgação científica. Porém, como indica d'Espagnat [40], ao tentar simplificar uma idéia complexa, estes textos terminam passando uma idéia incorreta sobre a Mecânica Quântica. É comum que, nestes casos, acabe-se passando a idéia, por exemplo, de que elétrons, prótons, fótons são bolinhas que colidem entre si, reforçando imagens clássicas, concepções essas "falsas, não no detalhe, mas de uma maneira essencial" [43:263]. São os professores que dão aula no nível médio que, inicialmente, devem tentar conseguir mudar este tipo de imagem. Claro que para isso eles devem ter uma idéia clara sobre os conceitos fundamentais. Isso, no entanto, não acontece. Durante o transcurso do trabalho de pesquisa, tivemos oportunidade de fazer um estudo exploratório com professores do ensino médio, pertencentes à rede pública e particular, em um curso de atualização em Física (Pró-Ciências FAPERGS-UFRGS 2000). Nessa ocasião, foi possível constatar que, efetivamente, os professores, embora motivados para incorporar temas de Mecânica Quântica em suas aulas e reconhecendo sua importância, muitas vezes não o fazem por sentirem-se despreparados para uma discussão conceitual sobre estes assuntos. $\mathrm{Na}$ prática, os cursos de formação de professores incluem, em geral, somente uma disciplina específica sobre os conceitos quânticos fundamentais, via de regra apresentada da maneira tradicional. Por outro lado, uma outra experiência-piloto, realizada com uma turma de estudantes do último curso do nível médio, nos permitiu verificar o interesse que esses temas despertam neles e a forma com que permitem discutir conceitos apresentados aos estudantes anteriormente. Para citar um exemplo, na apresentação do Princípio de Incerteza, os estudantes discutiram amplamente a questão da validade das leis da Física Clássica, embora não fossem inicialmente motivados para isto.

Embora uma formação mais completa dos professores seja um elemento fundamental para introduzir conceitos quânticos no nível médio, como apresentalos na realidade da escola brasileira exige mais estudo. Ou seja, ainda que nossa implementação didática tenha utilizado um mínimo de ferramentas matemáticas, para os estudantes do nível médio os elementos de Cálculo (e, obviamente, as equações diferenciais) são totalmente desconhecidos. Como fazer então para introduzir tais conteúdos sem cair em simplificações que levem a erros? Dever-se-á enfatizar, exclusivamente, a dimensão histórico-epistemológica da mudança introduzida pela Mecânica Quântica, como propõem diversos autores, ou será possível "aprofundar-se" um pouco mais nos aspectos conceituais, possivelmente fazendo uso de simulações computacionais para superar as deficiências matemáticas? São perguntas para as quais parece ainda não haver uma resposta clara, porém nosso trabalho sinaliza para esta segunda direção.

\section{References}

[1] L. McDermott e E. Redish, Am. J. Phys. 67(9), 755 (1999).

[2] I. M. Greca e M. A. Moreira, Inv. Ens. Ciên. 6(1), (2001).

[3] D. Zollman, NARST Annual Meeting, www.phys.ksu.edu/perg/papers/narst.html (1999).

[4] C.G. Hood, The Phys. Tea. 31(5), 290 (1993).

[5] L. Bao, P. Jolly e E. Redish, AAPT Summer meeting, www.physics.umd.edu/perg/cpt.html (1996).

[6] I. Johnston, K. Crawford e P. Fletcher, Int. Jour. Sci. Edu. 20(4), 427 (1998).

[7] B. Ambrose, P. Shaffer, R. Steinberg e L. McDermott, Am. J. Phys. 67(2), (1999).

[8] I. M. Greca e M. A. Moreira, Atas do II ENPEC, IFUFRGS, Porto Alegre (1999).

[9] P. Feyerabend, Contra o método, Ed. Verso, Lisboa (1993).

[10] P. Johnson-Laird, Mental Models, Harvard University Press, Cambridge (1983).

[11] M. A. Moreira, Inv. Ens. Ciên. 1(3) (1998).

[12] I. M. Greca e M. A. Moreira, Atas do VI EPEF, SBF, Florianópolis (1998).

[13] A. Zeilinger, Nature, 398(6724), 210 (1999).

[14] I. M. Greca e V. E. Herscovitz, Simp. David Bohm, 2, 85 (2001).

[15] M. Jammer, The philosophy of quantum mechanics, John Wiley, New York (1974).

[16] D. Bohm e B. Hiley, In Selleri (eds) Quantum mechanics versus local realism, Plenum, New York, 235 (1988).

[17] M. Paty, Eur. J. of Phys.20, 373 (1999).

[18] Z. Schreiber, The nine lives of Schrödinger's cat, University of London, London, Master thesis (1994).

[19] P. A. Dirac, The principles of quantum mechanics, Claredon Press, Oxford, 4a. edição (1930).

[20] R. Hughes, The structure and interpretation of quantum mechanics, Harvard University Press, Cambridge (1989).

[21] L. Davidovich, M. Brune, J. Raimond e S. Haroche, Phys. Rev. A, 53(3), 1295 (1996).

[22] W. H. Zurek, Phys. Today, 44(10), 36 (1991). 
[23] C. Gerry e P. Knight, Am. J. Phys. 65(10), 964 (1997).

[24] O. Pessoa Jr. Rev. Bras. Ens. Fis. 19(1), 27 (1997).

[25] V. B. Braginsky e F. Y. Khalili, Quantum measurement, Cambridge University Press, Cambridge (1992).

[26] A. Schenzle, Contemp. Phys, 37(4), 303 (1996).

[27] O. Pessoa Jr., Cad. Hist. Filos. Ciên. 2(2), 177 (1992).

[28] D. P. Ausubel, J. D. Novak e H. Hanesian, Psicologia educacional, Interamericana, Rio de Janeiro (1980).

[29] D. P. Ausubel, The acquisition and retention of knowledge. A cognitive view, Kluwer Academic Publishers, Dordrecht (2000).

[30] M. A. Moreira, Aprendizaje significativa, Editora da UnB, Brasilia (2000).

[31] R. P. Feynman, R. Leighton e M. Sands, The Feynman lectures on physics, Addison-Wesley Publishing Company, New York (1965).

[32] C. Cohen-Tannoudji, B. Diu e F. Laloe, Quantum mechanics, John Wiley, New York (1977).

[33] M. Nussenzveig, Curso de física básica, vol. 4, Edgard Blücher (1998).

[34] I. M. Greca e V. E. Herscovitz, Proceedings of the PHYTEB 2000, Barcelona (2000).

[35] I.M. Greca e V. E. Herscovitz, Atas do VII EPEF, SBF, Florianópolis (2000).

[36] S. C. Johnson, Psychometrika, 32(3), 241 (1967).

[37] A. Kearney e S. Kaplan, Env. and Behav., 29(5), 579 (1997).

[38] B. Garskof e J. Houston, Psy. Rev. 70(3), 277 (1963).

[39] M. A. Moreira e C. A. Santos, J. Res. Sci. Teach. 18(6), 525 (1981).

[40] E. Gussarsky e M. Gorodetsky, J. Res. Sci. Teach 25(5), 319 (1988).

[41] C. A. Santos e M. A. Moreira, Escalonamento multidimensional e análise de agrupamentos hierárquicos, Ed. da Universidade, Porto Alegre (1991).

[42] I. M. Greca, Construindo significados em mecânica quântica: resultados de uma proposta didática aplicada a estudantes de Física geral, IF-UFRGS, Porto Alegre, Tese de doutoramento (2000).

[43] B. d'Espagnat, Penser la science ou les enjeux du savoir, Gauthier-Villars, Paris (1990).

\section{Apêndice}

\section{COMO SABER SOBRE A EXIS- TENNCIA DE UM OBJETO SEM IN- TERAGIR COM ELE. (OU COMO É POSSÍVEL, NO MUNDO QUÂNTICO, VER NA ESCURIDÃO.)}

Um fabricante conseguiu inventar uma classe de bombas, cheias de um gás altamente combustível, que explodem quando são atingidas por um único fóton. Dadas essas características, o processo de produção deve ser realizado na escuridão. Depois de construídas as bombas, descobriu-se que por algum tipo de problema na fabricação, algumas estavam cheias e outras não, pois sobrou parte do gás combustível. Como se poderia saber quais eram as bombas "boas" (que funcionavam), sem perder toda a produção?

\section{Exercício}

1: Por que o fabricante perderia toda sua produção?

O fabricante, desesperado, se encontrou com um amigo, por acaso físico, que lhe disse ser possível salvar parte dessa produção. (Naturalmente o fabricante, antes de obter a sua ansiada resposta, teve que assistir a uma aula de Mecânica Quântica.) Vamos agora discutir qualitativamente o problema e depois fazer algumas contas.

Um dispositivo muito usado em Física, o interferômetro, que aparece na Fig. 1, permite separar um raio de luz incidente em dois, de modo que na saída do interferômetro é possível observar fenômenos de interferência. O dispositivo, que consta de 2 espelhos que refletem completamente a luz que chega até eles e 2 semiespelhos que transmitem uma parte dos raios que chegam até eles e refletem a outra parte, possui também dois detetores com alarmes na saída de forma que a "interferência construtiva" é detectada por um detector $D_{1}$, enquanto $D_{2}$ coleta a "interferência destrutiva". Imaginemos que a fonte só emite um fóton por vez e que o interferômetro é ajustado de tal modo que só toca o alarme do detector $D_{1}$, ou seja, só chegam fótons a este detector.

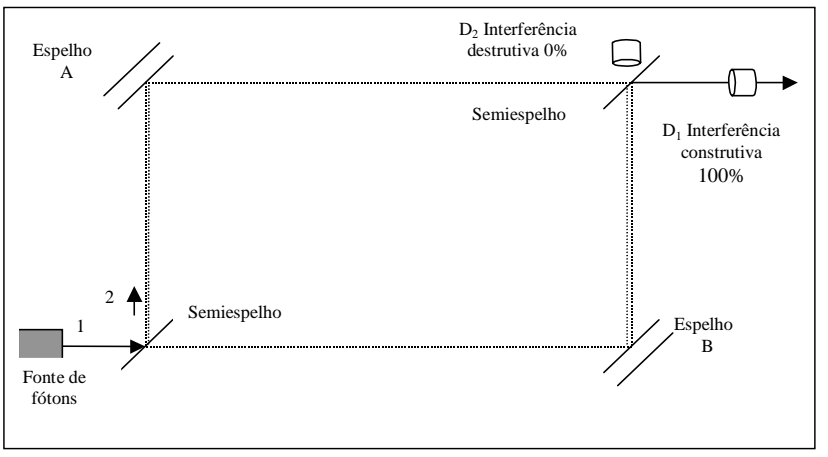

Figura 1

\section{Exercício}

2. Por que se obtém padrão de interferência para um fóton?

O que aconteceria se colocássemos um objeto bloqueando a passagem dos fótons por uma das direções? Os fótons que chegarem ao objeto (Fig. 2) são absorvidos, portanto se pode determinar por qual caminho passou cada fóton. Sabemos, pela experiência de Young, que se é possível determinar o caminho pelo qual passou um objeto quântico, não mais se obtém o padrão de 
interferência, existindo portanto probabilidades iguais de que o fóton seja detectado em cada um dos dois detetores.

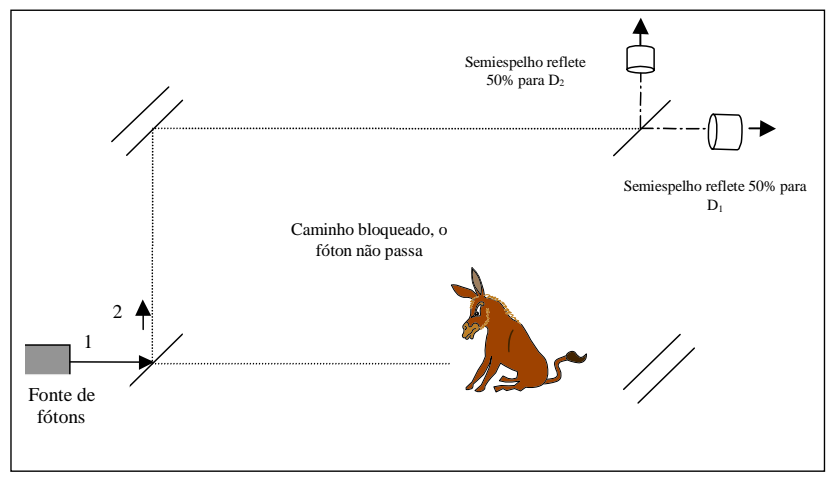

Figura 2

A interferência quântica acontece se não existe em princípio qualquer forma de determinar por qual dos caminhos alternativos o fóton (ou outro objeto quântico elementar) passou. Se, de alguma forma, podemos conhecer por onde passou, então a interferência desaparece.

Vejamos, agora, como auxiliar o nosso aflito fabricante com tal dispositivo. Coloquemos a bomba no interferômetro, bloqueando um dos caminhos. Se a bomba é ruim (ou seja, está sem gás, Fig. 3), é transparente e os fótons podem atravessá-la sem problemas e, portanto, soará apenas o alarme do detector D1, pois se produzirá interferência. Este caso é idêntico ao da Fig. 1.

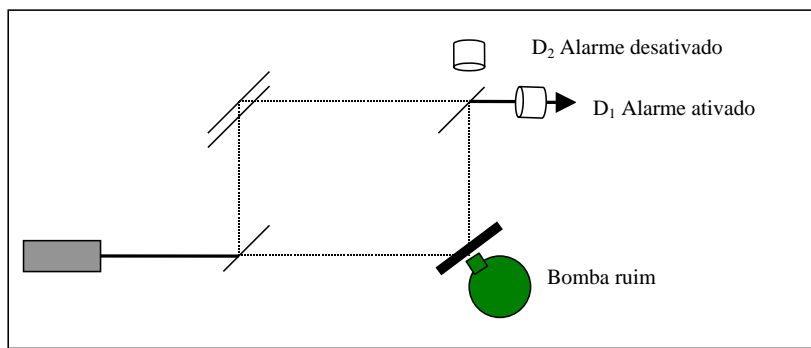

Figura 3

\section{Exercício}

3. Qual é a diferença entre as contribuições em $D_{1}$ para os casos apresentados nas Fig. 2 e 3 ?

Se a bomba, pelo contrário, é boa, i.e., está cheia de gás, pode absorver fótons e se poderia determinar, em princípio, o caminho escolhido pelos fótons. Portanto, não haverá padrão de interferência, podendo os fótons ser detectados tanto por $D_{1}$ como por $D_{2}$, aleatoriamente, pois o último semiespelho pode transmitir ou refletir aleatoriamente os fótons que chegam até ele, como acontece na Fig. 2.

\section{Exercício}

4. Por que não podem soar os alarmes dos dois detetores simultaneamente?

Neste caso, se se escuta o alarme de $D_{1}$ (Fig. 4), não se pode distinguir do caso anterior, em que a bomba era ruim. Se se escuta o alarme de $D_{2}$ (Fig. 5), sabe se que a bomba é boa. Se se escuta o Bang! saberemos que a bomba era boa....(Fig. 6)

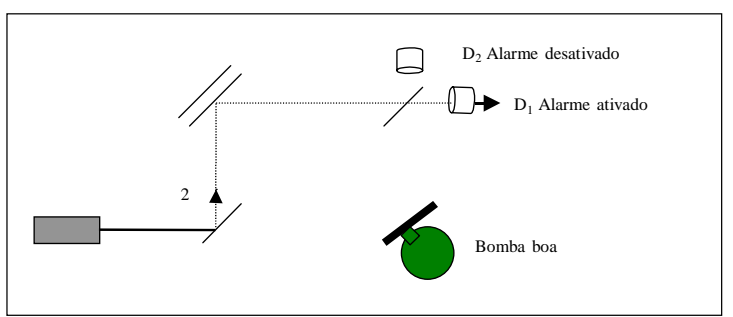

Figura 4

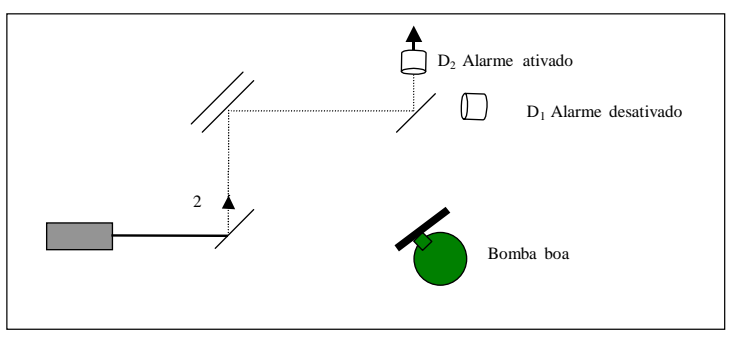

Figura 5

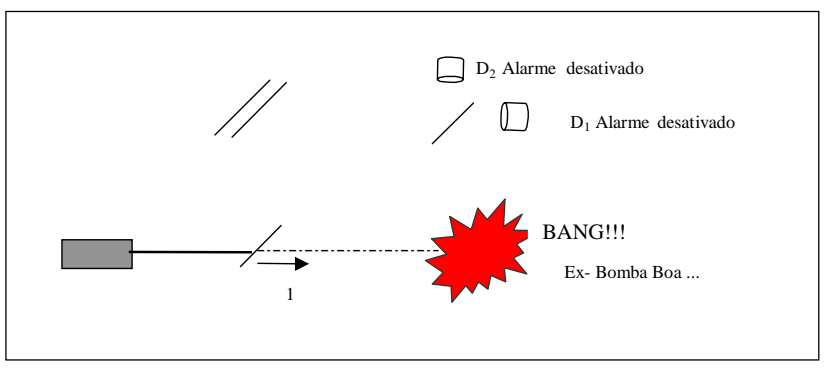

Figura 6

\section{Exercícios}

5. Por que se pode saber que a bomba está cheia de gás ao escutar o alarme de $D_{2}$ ?

6. Completar a seguinte planilha:

\begin{tabular}{|c|c|c|c|c|}
\hline $\begin{array}{c}\text { Estado } \\
\text { da Bomba }\end{array}$ & $\begin{array}{c}\text { Caminho } \\
\text { do fóton }\end{array}$ & $\begin{array}{c}\text { Padrão de } \\
\text { interfe } \\
\text { rência }\end{array}$ & $\begin{array}{c}\text { Observação } \\
\text { experimental }\end{array}$ & $\begin{array}{c}\text { Con- } \\
\text { clusão }\end{array}$ \\
\hline & & & & \\
\hline & & & & \\
\hline
\end{tabular}


No terceiro caso (Fig. 5), quando se ouve o detector $D_{2}$, consegue-se uma informação (que a bomba colocada estava cheia de gás) sem interagir com o objeto (pois, se o fóton tivesse interagido com a bomba, esta teria explodido). Ou seja, ainda que o fóton percorra o caminho onde não está a bomba, "tem-se" informação sobre o outro caminho que poderia ter escolhido. Ou seja, esta é uma "medida", sem o risco da interação com o objeto. Dispositivos que utilizam esses fundamentos poderiam, por exemplo, "fotografar" átomos, sem mudar seu estado. Experiências que mostram que isso pode ser feito na prática foram desenvolvidas na Universidade de Innsbruck, Áustria, em 1995.

\section{Exercícios}

7. Por que esta experiência não tem contrapartida no mundo clássico?

8. Determinar a probabilidade de que o alarme do detector $D_{1}$ dispare quando o recipiente da bomba está vazio, sabendo que:

$1>$ representa o fóton no caminho 1 ,

$2>$ representa o fóton no caminho 2 , que o espelho muda a direção do fóton (reflexão) segundo $\mid 1>\rightarrow$ $i \mid 2>$ e $|2>\rightarrow i| 1>$, que o semiespelho muda a direção do fóton (reflexão e transmissão) segundo e $\mid 1>\rightarrow(1 / 2)^{1 / 2}[|1>+i| 1>]$ e $\mid 2>\rightarrow(1 / 2)^{1 / 2}[\mid 2>$ $+i \mid 1>$ ]

9. Determinar a probabilidade de que a bomba esteja cheia de gás, sem interagir com ela.

Para o caso, então, de que se disponha de um dispositivo do tipo proposto aqui, existe uma probabilidade de $1 / 4$ de poder detectar as bombas boas. Podese mostrar que a probabilidade de "medições livres de interação" pode aproximar-se arbitrariamente de 1 , se se dispõe de um número grande de dispositivos.

\section{Exercício}

10. Quais propriedades dos objetos quânticos permitem fazer estas medições livres de interação?

\section{Bibliografia}

"Quantum bombing reality", de S. Adams, Physics Education, 1998.Vol. 33, n0 6, pp. 378-385. "Interactionfree measurement", de P. Kwiat, H. Weinfurt, T. Herzog, A. Zeilinger e M. Kasevich, Physical Review Letters, 1995. Vol. 74, n0 24, pp. 4763-4766. 